\title{
APROXIMACIÓN AL USO DE LAS REDES SOCIALES POR LAS FUERZAS Y CUERPOS DE SEGURIDAD EN ESPAÑA EN PERSPECTIVA INTERNACIONAL
}

\author{
APPROACH TO THE USE OF SOCIAL MEDIA BY SPANISH SECURITY FORCES \\ AND CORPS IN AN INTERNATIONAL PERSPECTIVE \\ Roberto Rodríguez Andrés y Julia María López-García \\ | rrodrigueza@comillas.edu | julia.Ig96@gmail.com | \\ Universidad Pontificia Comillas / Universidad de Navarra
}

\begin{abstract}
Resumen: Como parte de su política de comunicación, las fuerzas y cuerpos de seguridad de todo el mundo se han introducido en las redes sociales para relacionarse con los ciudadanos. A nivel internacional hay ya una amplia corriente de investigación sobre este tema, pero hasta el momento hay muy pocos estudios empíricos sobre el caso español, a pesar de que las policías de nuestro país son consideradas como un caso de éxito. El presente trabajo trata de realizar una primera aproximación sobre el uso que dan a estas redes sociales y analiza cuestiones como su compromiso con la comunicación 2.0, las comunidades que han conseguido generar y cuál es la actividad que desarrollan para difundir información e interaccionar con los ciudadanos. Ello se complementará con una comparación con los principales cuerpos policiales internacionales, lo que permitirá apuntar algunas claves que justifican el éxito español, así como futuras líneas de investigación a desarrollar en este campo. Palabras clave: policía; seguridad; redes sociales; comunicación 2.0; comunicación organizacional.
\end{abstract}


Abstract: As a part of their communication policy, security forces and corps around the world have been introduced into social networks to interact with citizens. At international level, there is a wide line of research on this subject, but there are very few empirical studies until now on the Spanish case, despite the fact that the police in our country is considered a case of success. In this paper we try to make a first approximation about the use they give to these social networks, analyzing issues such as their commitment to communication 2.0, the communities they have created and the activity that they develop to disseminate information and interact with the citizens. This will be complemented by a comparison with the main international police forces, which will allow us to point out some keys that justify Spanish success, as well as future lines of research to be developed in this field. Keywords: Police; Security; Social Networks; Communication 2.0; Organizational Communication.

\section{Introducción: Fuerzas y Cuerpos de Seguridad en España}

La seguridad es un principio básico consagrado en la Constitución española. En su artículo 17 se establece que «toda persona tiene derecho a la libertad y a la seguridad», una función asignada a las Fuerzas y Cuerpos de Seguridad, que tienen como misión, según el artículo 104, «proteger el libre ejercicio de los derechos y libertades y garantizar la seguridad ciudadana».

La regulación que afecta a estos organismos en nuestro país se desarrolló a través de la Ley Orgánica 2/1986, de 13 de marzo, de Fuerzas y Cuerpos de Seguridad. Concretamente en su artículo dos se estipula que estas están compuestas por las Fuerzas y Cuerpos de Seguridad del Estado, dependientes del Gobierno de la nación, los cuerpos de policía adscritos a las Comunidades Autónomas y los cuerpos de policía de las corporaciones locales:

$\rightarrow$ Las Fuerzas y Cuerpos de Seguridad del Estado, que ejercen sus funciones en todo el territorio nacional, están integradas por el Cuerpo Nacional de Policía, instituto armado de naturaleza civil cuyos orígenes se sitúan en 1824 y que actúa sobre todo en el ámbito urbano (Turrado, 2000) y la Guardia Civil, instituto armado de naturaleza militar fundado en 1844 y que despliega su actividad en el resto del territorio nacional y en el ámbito marítimo (Puig, 1984). Ambos cuerpos, en virtud de la Constitución, son los encargados de actuar también como Policía Judicial.

$\rightarrow$ Los Cuerpos de Policía dependientes de las Comunidades Autónomas, cuya constitución y regulación queda desarrollada en sus respectivos Estatutos de Autonomía (Pintado, 2016). Hasta el momento han desplegado 
esta competencia cuatro autonomías: Cataluña (Mossos d'Esquadra), País Vasco (Ertzaintza), Navarra (Policía Foral) y Canarias (Policía Canaria).

$\rightarrow$ Los Cuerpos de Policía dependientes de los ayuntamientos. Los más destacados en cuanto a número de agentes que los componen y población atendida son la Policía Municipal de Madrid, surgida en 1850, y la Guardia Urbana de Barcelona, en 1840, aunque con orígenes que se remontan al siglo XVIII (Olmos, 2016).

\section{El papel de la comunicación organizacional en las Fuerzas y Cuerpos de Seguridad}

La principal misión de estas fuerzas es velar por la seguridad. En ninguna de las normas que rigen su funcionamiento se encuentran referencias directas a cómo estos cuerpos pueden utilizar la comunicación para la consecución de sus objetivos, aunque en todas ellas se contemplan no sólo medidas activas sino también labores de prevención de la delincuencia, un apartado en el que la comunicación resulta fundamental.

Existe aún escasa bibliografía científica en nuestro país sobre las labores de comunicación de estas entidades, una línea que está ya más consolidada a nivel internacional (Motschall y Cao, 2002; Chermak y Weiss, 2005; Mawby, 2002 y 2010; Kingshott, 2011; Lee y McGovern, 2013 y 2014). Tomando como referencia las investigaciones de Cancelo (2004 y 2006), se puede afirmar que los cuerpos de seguridad españoles han entendido con el paso de los años que debían planificar su comunicación no sólo para que esta les ayudara en el cumplimiento de sus objetivos sino, también, para dar a conocer a la opinión pública las labores que llevan a cabo y buscar su respaldo y aprobación y, con ello, legitimación social.

Todo ello, además, y como continúa esta autora, en un contexto en el que podían ser juzgados sólo por las actuaciones polémicas que llevan a cabo, vinculadas a su función coactiva y de represión, que acaban trasladando una imagen negativa de ellos a la sociedad. Estos mismos objetivos están en la base de la denominada «comunicación estratégica» o stratcom en el ámbito de los ejércitos y la defensa, un campo en el que, sin embargo, sí se ha desarrollado ya una prolífica labor de investigación (Silvela, 2017).

Así, una de las primeras conclusiones a las que han ido llegando de forma progresiva los cuerpos policiales españoles es que debían abandonar los criterios de hermetismo y opacidad con los que actuaban en el pasado y que debían abrirse, como el resto de instituciones públicas en democracia, a una política de comunicación proactiva y de transparencia, habilitando departa- 
mentos específicos de comunicación dentro de sus organigramas. Algunos de estos departamentos, como el caso de la oficina de prensa de la Guardia Civil, fueron creados incluso ya en época del franquismo, concretamente en 1965, con la misión de mantener la relación con los medios de comunicación y dar a conocer sus actividades (Lorenzo, 2013). Esta estructura en el caso concreto de la Benemérita se ha ido reforzando en democracia, con la creación en ocasiones de delegaciones de prensa en provincias, sobre todo a partir de 1997 que es cuando se constituye su Oficina de Relaciones Informativas y Sociales (ORIS) (Fernández Vázquez, 2014). Y lo mismo ha ocurrido con la Policía Nacional, que creó su Oficina de Prensa en 1981, y con el resto de cuerpos autonómicos y locales (Cancelo, 2004).

Estas estructuras comunicativas pretenden evitar que las noticias que se generan sobre su actividad en los medios se centren sólo en sus funciones represivas, buscando trasladar a la opinión pública otros cometidos que llevan a cabo y que les pueden generar una imagen positiva, especialmente en el campo de la protección de los ciudadanos. Es el caso, por ejemplo, de la lucha contra el terrorismo, un asunto que les ha dado especial visibilidad y apoyo social, entre otras cosas porque además de proteger a la sociedad, han sido uno de los objetivos prioritarios de los terroristas, lo que ha generado una corriente de solidaridad pública hacia ellos (Rodrigo, 1991; Cuesta, Canel y Gurrionero, 2012).

Y la realidad, tal como se ha podido constatar en distintos sondeos del Centro de Investigaciones Sociológicas (CIS) y en estudios de otras empresas demoscópicas, es que en nuestra reciente historia democrática, las Fuerzas y Cuerpos de Seguridad han conseguido este objetivo, puesto que han mantenido de forma estable una alta valoración por parte de los ciudadanos, salvándose así del descrédito general que ha ido afectando a otras instituciones del Estado (Justel, 1982; Díez Nicolás, 1989 y 2012; Raldúa, 1996; Blanco, 2017; Sáinz, 2018; Lucas-Torres, 2019). En estos estudios se ha ido comprobando cómo la Guardia Civil y la Policía, junto con las Fuerzas Armadas, han sido las únicas instituciones que nunca han suspendido en valoración popular desde 1987 (primer año que se preguntó por ellas) hasta la actualidad. Hasta 2013, su valoración solo estaba por debajo de la de la Monarquía; sin embargo, a partir de ese año la han superado, tendencia que se mantiene en las últimas encuestas.

\section{Cuerpos de Seguridad en las redes sociales: objetivos y metodología de la investigación}

En esta apuesta por la comunicación, las Fuerzas y Cuerpos de Seguridad, tanto nacionales como autonómicas y locales, no fueron ajenas a la llegada de Internet 
y, posteriormente, de las redes sociales y la comunicación 2.0 (Alcázar, 2001). Estas herramientas supusieron un avance a la hora de encontrar nuevas vías de relación y comunicación con los ciudadanos, más allá de la vía tradicional centrada en las relaciones con los medios, y potenciando la bidireccionalidad, el diálogo y la transparencia, todo ello con el objetivo de mejorar su reputación. Como afirman Moya y Blanco (2015: 149), «las fuerzas y cuerpos de seguridad encontraron en los medios sociales una herramienta tanto a nivel estratégico como operativo, destacando las posibilidades de colaboración policial y las situaciones de emergencia».

En este campo, y siguiendo el clásico esquema de Mergel (2013), se considera que hay tres etapas que marcan el uso de las redes sociales por parte de las administraciones públicas: «representativa» (caracterizada por la simple difusión de mensajes), «comprometida» (que implica responder e interactuar con las audiencias) y «colaborativa» (propiciando que los ciudadanos participen y se impliquen en la actividad de la institución).

¿En qué fase de este esquema se encuentran las fuerzas y cuerpos de seguridad españoles? ¿Cuál es el uso que están haciendo de las redes sociales con el objetivo de reforzar la relación con los ciudadanos? ¿Están contribuyendo estas herramientas a afianzar su buena valoración entre la opinión pública? Estos serán los principales objetivos de la presente investigación, que se centrará de forma prioritaria en el estudio de las entidades policiales de ámbito nacional (Policía Nacional y Guardia Civil) así como en los cuatro cuerpos de policía autonómicos (Mossos d'Esquadra, Ertzaintza, Policía Foral de Navarra y Policía Canaria), aunque con menciones también a las policías municipales de Madrid y Barcelona, por ser los cuerpos policiales de las dos ciudades más pobladas de España y, por ello, con mayor repercusión pública.

Para tratar de obtener datos más representativos y que permitan valorar en mejor medida su actividad en redes se realizará, asimismo, una comparación entre su presencia y actividad en estas plataformas con la que tienen otras instituciones públicas en nuestro país (basándonos en estudios precedentes sobre esta materia), así como con distintos cuerpos de seguridad internacionales.

En los últimos años, la comunidad académica de distintos países ha realizado estudios individualizados acerca de la actividad en redes de sus cuerpos policiales, con un buen número de investigaciones (detalladas en la tabla 1), la gran mayoría centradas exclusivamente en Twitter. Pero, hasta el momento, no se ha llevado a cabo un análisis comparativo con perspectiva internacional y tomando como referencia el conjunto de las principales redes sociales. Para acometer esta comparación se han seleccionado también como muestra de análisis las policías más representativas de los países del 
G7 (Alemania, Canadá, Estados Unidos, Francia, Italia, Japón y Reino Unido) y de las naciones iberoamericanas presentes en el G20 (Argentina, Brasil y México), así como organismos de coordinación policial supranacionales (Interpol y Europol).

Tabla 1. Investigaciones realizadas sobre el uso de las redes sociales por parte de cuerpos policiales.

\begin{tabular}{|l|l|}
\hline País & Investigaciones \\
\hline Estados Unidos & $\begin{array}{l}\text { Heverin y Zach, 2011 y 2012; Lieberman, Koetzle y Sakiyama, 2013; Brainard y Edlins, } \\
\text { 2014; Davis, Alves y Sklansky, 2014; Edlins y Brainard, 2016; Dai et al., 2017. }\end{array}$ \\
\hline Reino Unido & Crump, 2012; Procter, 2013; Bullock, 2018. \\
\hline Canadá & $\begin{array}{l}\text { Trottier, 2012; Ruddell y Jones, 2013; Carpentier-Laberge, 2015; Schneider, 2016; } \\
\text { Schneider y Altheide, 2016; O'Connor, 2017; Kudla y Parnaby, 2018. }\end{array}$ \\
\hline Holanda & $\begin{array}{l}\text { Van de Velde, Meijer y Homburg, 2015; Grimmelikhuijsen y Meijer, 2015; Meijer y } \\
\text { Torenvlied, 2016. }\end{array}$ \\
\hline Australia & Kelly y Finlayson, 2015. \\
\hline Francia & Wybo et al., 2014. \\
\hline
\end{tabular}

Para todos los casos, se ha adoptado una metodología basada en la observación y seguimiento empírico de los perfiles en redes de estas entidades, y se toma como periodo de estudio el comprendido entre el 1 de enero y el 31 de diciembre de 2018, complementado con el uso de herramientas de monitorización como Fanpage Karma (www.fanpagekarma.com) y Twitonomy (www.twitonomy.com).

Se ha analizado, en primer lugar, el compromiso inicial mostrado con estas nuevas herramientas y el entorno de la comunicación 2.0, medido en la fecha en la que se incorporaron a ellas y cómo han ido ampliando su presencia en las mismas a lo largo del tiempo. A continuación, se cuantificarán las comunidades que han conseguido crear, a través del número de seguidores con el que cuentan. Por último, se analizará también la actividad que generan en las mismas (número y frecuencia de publicaciones) y la repercusión que consiguen en términos de reacciones y de fomento del diálogo con los ciudadanos.

Se trata, por tanto, de un análisis eminentemente cuantitativo, que se plantea como una primera aproximación para conocer si las policías españolas están aprovechando de forma adecuada las potencialidades comunicativas que les brindan estas plataformas para mejorar su relación con los ciudadanos y seguir reforzando su buena valoración pública, al tiempo que nos permitirá describir si su compromiso en este campo es mayor o menor que el de otros cuerpos policiales internacionales. 


\section{Presencia en las redes sociales y comunidades generadas}

A 31 de diciembre de 2018, como puede apreciarse en la tabla 2, el conjunto de Fuerzas y Cuerpos de Seguridad españoles analizados tenían un total de 30 perfiles abiertos en redes sociales. Los que cuentan con mayor presencia son la Guardia Civil y los Mossos d'Esquadra (6 cada uno), seguidos de la Policía Nacional (con 5), Policía Foral de Navarra y Policía Municipal de Madrid (4), Guardia Urbana de Barcelona (3) y, por último, Ertzaintza y Policía Canaria (con una única red).

Por tipo de plataforma, la más generalizada es Twitter, en la que tienen cuenta todos ellos (la Policía Nacional, incluso, con dos perfiles, uno en español y otro en inglés), seguida de Facebook, Instagram, YouTube y Flickr. La presencia en otras redes es testimonial, como el caso de la Guardia Civil en Tumblr y de los Mossos en SlideShare. Además, algunas de ellas han sido ya cerradas y, por tanto, no introducidas en este estudio, como el canal conjunto en Tuenti que abrieron en 2010 Policía y Guardia Civil y que cesó su actividad a finales de 2017 al desaparecer esta red social juvenil.

Tabla 2. Presencia de las Fuerzas y Cuerpos de Seguridad españoles en redes sociales.

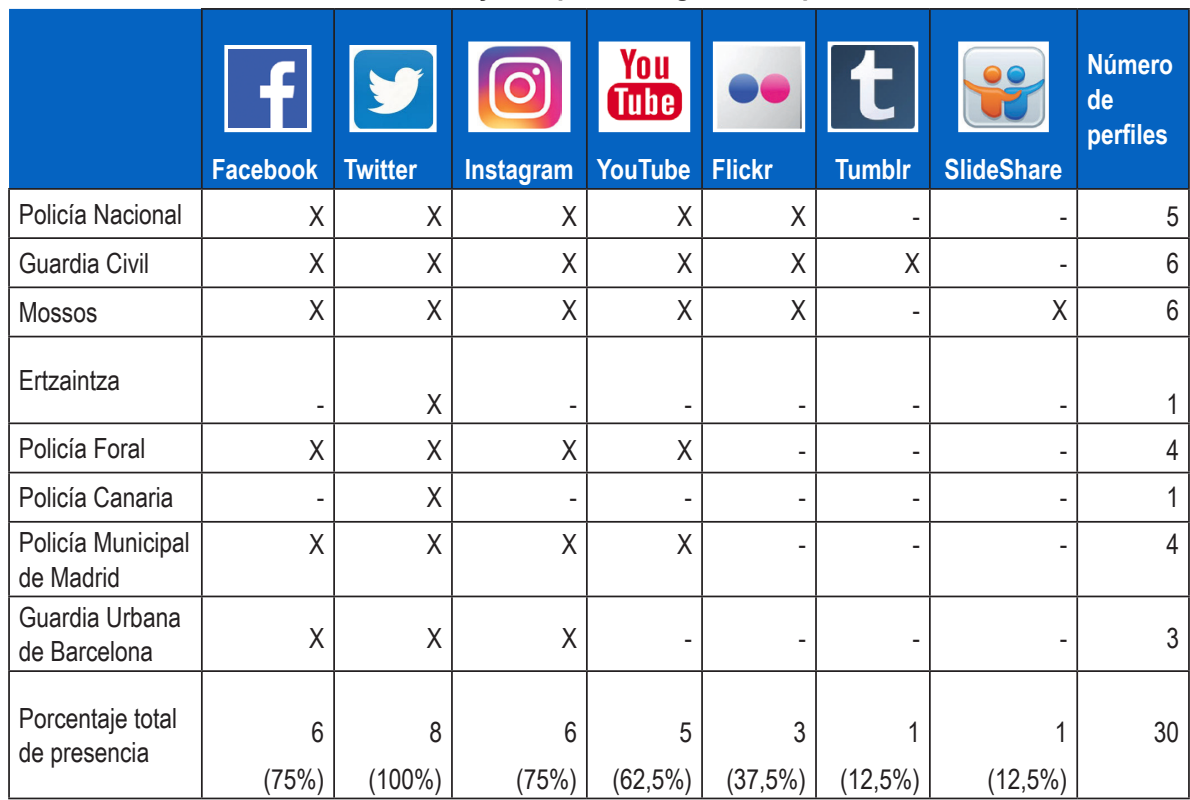

Fuente: elaboración propia a partir de consulta a las redes sociales (fecha de consulta: 31/12/2018).

Se aprecia, por tanto, un compromiso bastante generalizado de los cuerpos de seguridad españoles con la participación en redes sociales como herramienta de comunicación y difusión de sus actividades. Este compromiso, sin embar- 
go, no resulta un hecho diferencial con respecto a la realidad de otros países, puesto que, como puede apreciarse en la tabla 3, la presencia en redes es una experiencia bastante común en este tipo de entidades. En todos los casos, hay coincidencia también en apostar claramente por Facebook, Twitter, Instagram y YouTube como las plataformas principales, aunque a nivel internacional se da más relevancia a LinkedIn, una red no utilizada por las policías españolas.

Tabla 3. Presencia de los cuerpos de seguridad internacionales en redes sociales.

\begin{tabular}{|c|c|c|c|c|c|c|c|c|c|}
\hline País/región & Cuerpo policial & 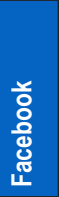 & 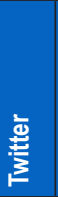 & 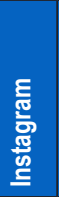 & 을 & $\frac{\text { 늠 }}{\frac{0}{1}}$ & $\begin{array}{l}\text { 등 } \\
\text { 들 }\end{array}$ & $\frac{9}{\frac{5}{6}}$ & $\begin{array}{l}\text { Número } \\
\text { de } \\
\text { perfiles }\end{array}$ \\
\hline Internacional & Interpol & $\mathrm{X}$ & $X$ & $\mathrm{X}$ & $x$ & - & $X$ & - & 5 \\
\hline UE & Europol & $x$ & $x$ & $x$ & $x$ & - & $x$ & - & 5 \\
\hline \multirow[t]{2}{*}{ USA } & FBI (Federal Bureau of Investigation) & $\mathrm{X}$ & $X$ & $\mathrm{X}$ & $X$ & $X$ & $X$ & - & 6 \\
\hline & NYPD (New York Police Dpt.) & $x$ & $x$ & $\mathrm{X}$ & $x$ & - & - & - & 4 \\
\hline \multirow[t]{2}{*}{ Francia } & Gendarmerie & $x$ & $x$ & $x$ & $x$ & - & $x$ & - & 5 \\
\hline & Police Nationale & $x$ & $x$ & $x$ & $x$ & - & - & - & 4 \\
\hline Italia & Polizia di Stato & $\mathrm{X}$ & $x$ & $\mathrm{X}$ & $X$ & - & - & $\begin{array}{r}X \\
\text { (1) }\end{array}$ & 5 \\
\hline Alemania & Bundespolizei & $x$ & $x$ & $x$ & $x$ & - & - & - & 4 \\
\hline \multirow[t]{2}{*}{ Argentina } & Policía Federal & $x$ & $x$ & $x$ & $x$ & - & - & - & 4 \\
\hline & Gendarmería Nacional & $x$ & $x$ & $x$ & $x$ & $x$ & - & - & 5 \\
\hline Australia & Australian Federal Police & $\mathrm{X}$ & $x$ & $x$ & $\mathrm{X}$ & - & - & - & 4 \\
\hline Brasil & Policía Federal & $x$ & $x$ & $x$ & $x$ & $x$ & - & $\begin{array}{r}X \\
(2) \\
\end{array}$ & 6 \\
\hline Canadá & Royal Canadian Mounted Police & $x$ & $x$ & $x$ & $x$ & - & - & - & 4 \\
\hline Reino Unido & Scotland Yard & $x$ & $x$ & $x$ & $x$ & - & - & - & 4 \\
\hline Japón & National Police Agency & $\mathrm{X}$ & $x$ & - & - & - & - & - & 2 \\
\hline México & Policía Federal & $x$ & $x$ & $x$ & $x$ & - & - & - & 4 \\
\hline $\mathrm{N}^{0}$ total & & & & & & & & & 71 \\
\hline
\end{tabular}

Fuente: elaboración propia a partir de consulta a las redes sociales (fecha de consulta: 31/12/2018).

(1) Telegram

(2) Soundcloud

Pero si la situación en la actualidad no arroja diferencias significativas, la perspectiva histórica aporta un primer dato relevante a efectos de esta investigación, y es que las policías españolas estuvieron entre las pioneras a la hora de introducirse en este ámbito a nivel internacional, tal como puede apreciarse en la tabla 4. 
Tabla 4. Ranking de policías por orden de creación de sus perfiles en redes sociales.

\begin{tabular}{|c|c|c|c|}
\hline Youtube & Twitter & Facebook & Instagram \\
\hline $\begin{array}{l}\text { - FBI USA (oct. 05). } \\
\text { - Policía Nacional ESP } \\
\text { (ene. 06). } \\
\text { - Guardia Civil ESP } \\
\text { (jun. 06). } \\
\text { - Polizia di Stato IT (feb. } \\
\text { 07). } \\
\text { - NYPD USA (nov. 07). } \\
\text { - Scotland Yard RU (jul. } \\
\text { 09). } \\
\text { - Policía Montada CAN } \\
\text { (oct. 09). } \\
\text { - Mossos ESP (feb. 10) } \\
\text { - Interpol (sep. 10). } \\
\text { - Policía Federal ARG } \\
\text { (feb. 11). } \\
\text { - Policía Federal BR } \\
\text { (may. 11). } \\
\text { - Europol (dic. 12). } \\
\text { - Policía Foral ESP } \\
\text { (jul. 13). } \\
\text { - Gendarmerie FR (oct. } \\
\text { 13). } \\
\text { - Police Nationale FR } \\
\text { (feb. 14). } \\
\text { - Australian Federal } \\
\text { Police (feb. 14). } \\
\text { (enelicía Municipal de } \\
\text { (jul. 14). } \\
\text { - Gendarid ESP (abr. 14). } \\
\text { - Pundespolizei ALE Federal MEX } \\
\text { (ago. 14). }\end{array}$ & 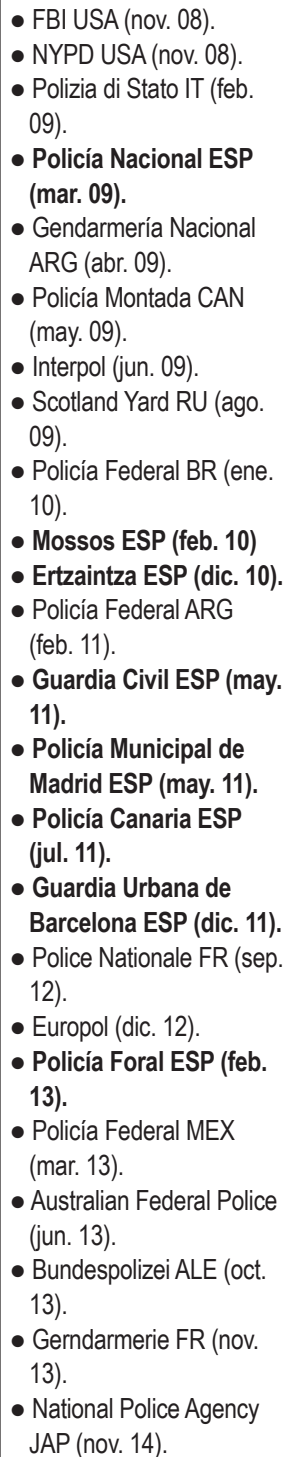 & 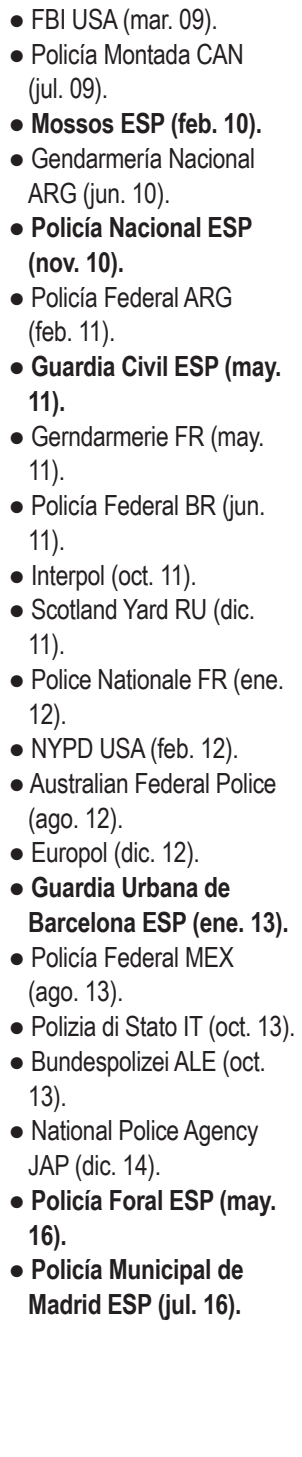 & 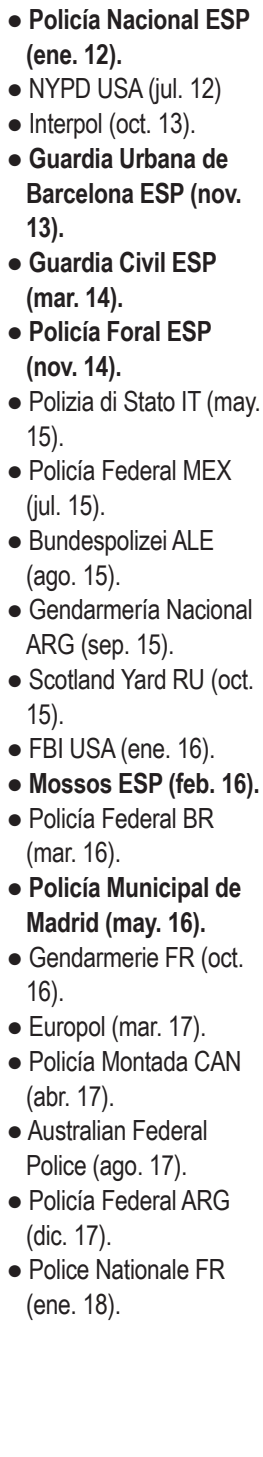 \\
\hline
\end{tabular}

Fuente: elaboración propia a partir de consulta a las redes sociales (fecha de consulta: 31/12/2018).

La Policía Nacional y la Guardia Civil fueron el segundo y tercer cuerpo policial, respectivamente, en abrir perfil en YouTube, tan sólo superadas 
por el FBI que, por otra parte, es el que primero abrió cuenta en 3 de las 4 redes principales. En el caso de Twitter y Facebook, la Policía Nacional se sitúa también en el ranking de los cinco primeros perfiles abiertos (en $4^{\mathrm{a}}$ y $5^{\mathrm{a}}$ posición, respectivamente) y, en esta última red, destacan también los Mossos d'Esquadra en $3^{\mathrm{a}}$ posición.

Pero aún es más significativo el carácter pionero de las policías españolas en Instagram, última de las grandes redes incorporadas al espacio 2.0, puesto que entre los seis primeros cuerpos policiales que abrieron cuenta hay cinco policías españolas, siendo la Policía Nacional la primera que se introdujo en esta red de entre todas las analizadas.

Este carácter pionero a la hora de apostar por las redes sociales se comprueba también si se hace una comparación con otras instituciones públicas en nuestro país. En el caso de los ministerios del Gobierno español, tal como ha descrito Rodríguez (2017), la primera cuenta no se abrió hasta julio de 2007 (la del Ministerio de Defensa en YouTube), año y medio después que la de la Policía Nacional. Incluso se adelantaron al lanzamiento de la primera cuenta oficial de Moncloa, también en YouTube, que fue en diciembre de 2006. Y lo mismo puede comprobarse en la fecha de incorporación a Instagram, puesto que los cuerpos de seguridad abrieron sus perfiles ya desde 2012 y 2013 y, en el caso de los ministerios, no lo harían hasta el año 2016.

La situación actual y la perspectiva histórica comparada a nivel internacional permiten comprobar el compromiso de los cuerpos policiales españoles por la comunicación 2.0 a través de las redes. Pero esta primera parte del análisis debe incluir también una referencia a la dimensión de las comunidades que han logrado generar en las mismas, medidas en términos del número de seguidores con los que cuentan y, por tanto, público al que alcanzan con sus mensajes.

De entre todas las redes, es Twitter en la que se produce mayor seguimiento. A 31 de diciembre de 2018, como se detalla en la tabla 5, la cuenta de la Policía Nacional en esta red social (@policia) acumulaba 3,17 millones de seguidores, figurando así entre las 100 cuentas españolas más seguidas, posición que viene repitiendo desde hace años (Twitter, 2019). También es destacable el número de seguidores del perfil de la Guardia Civil (@guardiacivil), que suma 1,27 millones. Por establecer una comparación que ayude a comprender mejor la dimensión de estas cifras, la cuenta oficial del Gobierno en esta red (@desdelamoncloa) no supera los 572.000 seguidores. Y desde una perspectiva internacional, se observa que las policías españolas están en las posiciones más elevadas del ranking. Ante esto, y como afirma Padilla (2016), se puede hablar de que las cuentas en Twitter de los cuerpos de segu- 
ridad españoles «se han convertido en arquetipo de éxito de redes sociales institucionales en España y en el extranjero» (2016: 88).

Tabla 5. Ranking de policías por número de seguidores en redes sociales.

\begin{tabular}{|c|c|c|}
\hline Twitter & Facebook & Instagram \\
\hline $\begin{array}{l}\text { - Policía Nacional ESP, 3,17 millones. } \\
\text { - FBI USA, 2,42 millones. } \\
\text { - Guardia Civil ESP, 1,27 millones. } \\
\text { - Policía Federal BR, 1,24 millones. } \\
\text { - Scotland Yard RU, 1,21 millones. } \\
\text { - NYPD USA, 602.000. } \\
\text { - Mossos ESP, 513.000. } \\
\text { - Police Nationale FR, } 450.000 . \\
\text { - Gendarmerie FR, 423.000. } \\
\text { - Policía Municipal Madrid ESP, } \\
\text { 370.000. } \\
\text { - Policía Federal MEX, } 351.000 . \\
\text { - Policía Montada CAN, } 223.000 . \\
\text { - National Police Agency JAP, } 220.000 . \\
\text { - Bundespolizei ALE, } 190.000 . \\
\text { - Interpol, } 149.000 . \\
\text { - Policía Federal ARG, } 112.000 . \\
\text { - Gendarmería ARG, } 85.900 . \\
\text { - Polizia di Stato IT, 81.800. } \\
\text { - Europol, 81.500. } \\
\text { - Guardia Urbana Barcelona ESP, } \\
\text { 53.000. } \\
\text { - Australian Federal Police, } 26.100 . \\
\text { - Ertzaintza ESP, 25.400. } \\
\text { - Policía Foral ESP, 23.900. } \\
\text { - Policía Canaria ESP, 8.328. }\end{array}$ & $\begin{array}{l}\text { - Policía Federal BR, } 2,74 \text { millones. } \\
\text { - FBI USA, 2,37 millones. } \\
\text { - Policía Federal MEX, } 918.000 . \\
\text { - NYPD USA, 820.000. } \\
\text { - Gendarmerie FR, 750.000. } \\
\text { - Policía Nacional ESP, } 700.000 . \\
\text { - Police Nationale FR, 689.000. } \\
\text { - Polizia di Stato IT, 464.000. } \\
\text { - Australian Federal Police, } 416.000 . \\
\text { - Mossos ESP, 335.000. } \\
\text { - Policía Federal ARG, 305.000. } \\
\text { - Guardia Civil ESP, 270.000. } \\
\text { - Gendarmería ARG, } 143.000 . \\
\text { - Policía Montada CAN, } 219.000 . \\
\text { - Scotland Yard RU, } 211.000 . \\
\text { - Interpol, 124.000. } \\
\text { - Bundespolizei ALE, 77.000. } \\
\text { - Europol, 61.500. } \\
\text { - Guardia Urbana Barcelona ESP, } \\
\text { 16.000. } \\
\text { - Policía Municipal Madrid ESP, } \\
\text { 12.000. } \\
\text { - National Police Agency JAP, } 2.000 . \\
\text { - Policía Foral ESP, 1.800. }\end{array}$ & $\begin{array}{l}\text { - Policía Federal BR, } 653.000 . \\
\text { - Policía Nacional ESP, } \\
390.000 . \\
\text { - NYDP USA, } 331.000 . \\
\text { - Guardia Civil ESP, } 184.000 . \\
\text { - FBI USA, } 182.000 . \\
\text { - Polizia di Stato IT, } 125.000 . \\
\text { - Mossos ESP, 115.000. } \\
\text { - Gendarmerie FR, 85.900. } \\
\text { - Bundespolizei ALE, } 70.500 . \\
\text { - Gendarmería ARG, } 42.500 . \\
\text { - Scotland Yard RU, } 39.800 . \\
\text { - Police Nationale FR, } 39.200 . \\
\text { - Policía Federal MEX, } 26.500 . \\
\text { - Australian Federal Police, } \\
\text { 24.300. } \\
\text { - Policía Federal ARG, } 22.200 . \\
\text { - Policía Montada CAN, } 19.700 . \\
\text { - Interpol, } 18.700 . \\
\text { - Guardia Urbana Barcelona } \\
\text { ESP, } 14.000 . \\
\text { - Policía Municipal Madrid } \\
\text { ESP, } 11.000 . \\
\text { - Europol, } 10.500 . \\
\text { - Policía Foral ESP, 1.700. }\end{array}$ \\
\hline
\end{tabular}

Fuente: elaboración propia a partir de consulta a las redes sociales (fecha de consulta: 31/12/2018).

Esta posición de liderazgo en Twitter se repite también en Instagram, donde tanto la Policía Nacional como la Guardia Civil se colocan entre las cinco cuentas más seguidas y la de los Mossos d'Esquadra en $7^{\mathrm{a}}$ posición, aunque es inferior en Facebook. Si se suma el número total de seguidores de todos los cuerpos policiales, el ranking está encabezado por el FBI (con 4,97 millones) seguido de la Policía Federal de Brasil (4,63 millones) y la Policía Nacional española (4,26 millones); aunque si se aplicara proporcionalidad con respecto a la población total de cada una de estas naciones, las cifras de la policía española adquirirían mucha mayor relevancia, lo que aplicaría igualmente al resto de cuerpos policiales de nuestro país. 


\section{Actividad de las Fuerzas y Cuerpos de Seguridad en las redes sociales}

De todas las cifras apuntadas en el epígrafe anterior, se puede constatar que las redes sociales de nuestras fuerzas y cuerpos de seguridad han conseguido captar la atención del público, hasta situarlas en primeras posiciones a nivel mundial en número de seguidores.

¿A qué se debe este hecho? ¿Qué labor han realizado en la comunicación 2.0 con los ciudadanos para atraer su atención? Estas preguntas nos introducen en la segunda parte de este análisis, más centrada en describir cuál es la actividad comunicativa que llevan a cabo, es decir, si utilizan estas plataformas de forma adecuada para fomentar la relación con los ciudadanos.

La primera consideración a este respecto es que, en contra de lo que ha sido habitual en otras instituciones públicas, la práctica totalidad de los perfiles abiertos están activos, es decir, publican mensajes de forma periódica y no han sido abandonados con el tiempo. De todos ellos, tan sólo las cuentas de Flickr de la Policía Nacional y la Guardia Civil permanecen inactivas desde hace más de cinco años, lo que puede explicarse a tenor de que esta red social especializada en la difusión de fotografías ha sido abandonada progresivamente en favor de Instagram.

En el caso de Twitter, la red en la que tienen mayor número de seguidores, la media de mensajes diarios que publican los cuerpos de seguridad españoles se sitúa en 8,39 , mientras que la de las entidades internacionales analizadas está en 5,01, lo que da cuenta del mayor uso que hacen de esta red en su comunicación con los ciudadanos. De entre todas las policías, como se apunta en la tabla 6, la Guardia Civil ocupa la primera posición (con 18,18 tuits diarios), quedando la Policía Nacional en cuarto puesto (con 10,98). Esta frecuencia de publicación es muy superior también a la que se registra en otras administraciones públicas españolas. Por ejemplo, la media de los 17 parlamentos autonómicos está en 4,55 (Rodríguez y Álvarez, 2018) y, en el caso de los ministerios del Gobierno, es de 4,03 (Rodríguez, 2017).

Pero siendo este indicador importante, podría generarse la confusión de que bastaría con publicar muchos mensajes para ser considerado un perfil exitoso en Twitter, lo cual no debe entenderse así, ya que de nada serviría si nadie los atiende. 
Tabla 6. Promedio de mensajes diarios publicados en Twitter.

\begin{tabular}{|l|r|l|r|l|r|}
\hline Guardia Civil & 18,18 & Policía Federal ARG & 6,97 & Interpol & 3,16 \\
\hline Policía Federal MEX & 15,17 & Police Nationale FR & 6,31 & FBI USA & 3,05 \\
\hline Scotland Yard RU & 12,08 & Ertzaintza & 5,99 & Europol & 2,35 \\
\hline Policía Nacional & 10,98 & Policía Foral & 5,15 & Australian Federal Police & 1,88 \\
\hline NYPD USA & 9,36 & Polizia di Stato IT & 4,47 & Policía Montada CAN & 1,84 \\
\hline Policía Municipal Madrid & 9,01 & Gendarmerie FR & 4,44 & Gendarmería Nacional ARG & 0,95 \\
\hline Mossos & 8,84 & Policía Federal BR & 3,94 & National Police Agency JAP & 0,95 \\
\hline $\begin{array}{l}\text { Guardia Urbana } \\
\text { Barcelona }\end{array}$ & 8,1 & Bundespolizei ALE & 3,27 & Policía Canaria & 0,91 \\
\hline
\end{tabular}

Fuente: elaboración propia a partir de la monitorización de perfiles en Twitonomy (del 1/01/2018 al 31/12/2018).

A este respecto, ya se ha apuntado que estas cuentas acumulan muchos seguidores, lo que es una muestra de que hay interés por lo que publican. Pero esta realidad se reafirma al analizar el porcentaje de sus tuits que son retuiteados por sus seguidores al considerarlos relevantes y dignos de ser compartidos. De nuevo, la Policía Nacional y la Guardia Civil, así como los Mossos d'Esquadra, ocupan posiciones de cabeza entre todas las policías analizadas, como se refleja en el gráfico 1. Y un buen ejemplo de ello es el hecho de que un tuit de la Policía Nacional fuera el más retuiteado en España en 2017 (concretamente el publicado el 17 de agosto sobre los atentados en Barcelona, con más de 100.000 retuits), según informó la propia red social.

Gráfico 1. Porcentaje de tuits que son retuiteados en cada perfil.

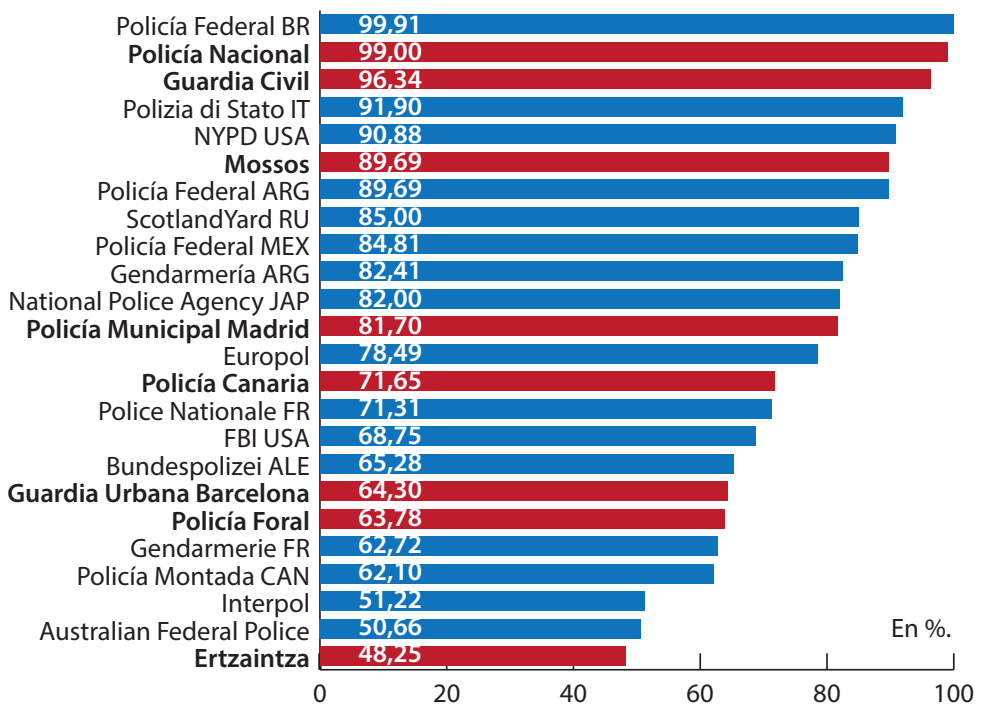

Fuente: elaboración propia a partir de la monitorización de perfiles en Twitonomy (del 1/01/2018 al 31/12/2018). 
Cifras similares a estas pueden encontrarse también en Facebook, la segunda red social en público total. En número de mensajes diarios, el ranking está encabezado por la Policía Federal de México (con 9,3), seguido de la Policía Federal de Argentina (6). En tercer y cuarto puesto aparecen los Mossos y la Policía Nacional (con 4,9 y 3,2, respectivamente) y, tras ellos, figura el FBI (con 3). En este caso, la respuesta de los usuarios a estas cuentas puede medirse en función del grado de compromiso o engagement, un indicador que la herramienta de monitorización Fanpage Karma obtiene tras dividir el número diario de likes, comentarios y publicaciones compartidas entre el número total de seguidores de cada cuenta.

Siguiendo este parámetro, y tal como se muestra en el gráfico 2, se observa que el nivel de compromiso de los usuarios es más bajo que en Twitter, pero una vez más se comprueba que los perfiles de las policías españolas se sitúan a la cabeza a nivel internacional, con una posición destacada para los Mossos d'Esquadra (1,47 por ciento), Guardia Civil (1 por ciento) y Policía Nacional $(0,99$ por ciento).

Gráfico 2. Compromiso de los usuarios con las cuentas en Facebook.

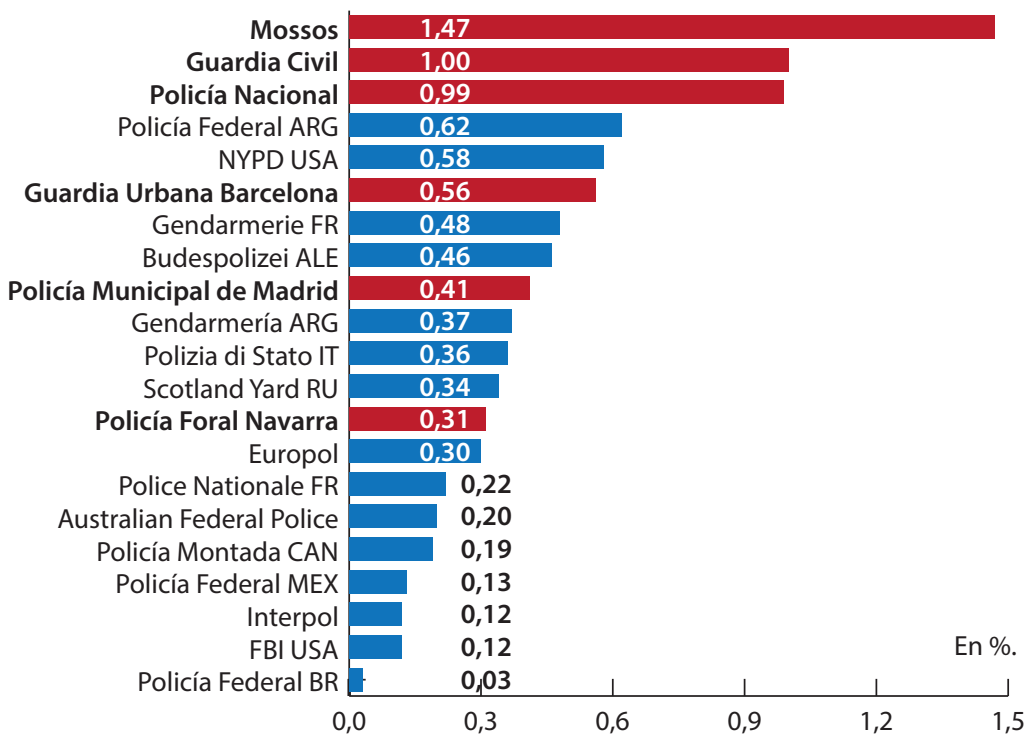

Fuente: elaboración propia a partir de la monitorización de perfiles en Fanpage Karma (del 1/01/2018 al 31/12/2018).

Hay, sin embargo, un aspecto en el que las cuentas policiales aún presentan margen de mejora en su compromiso con la comunicación 2.0. Nos referimos 
al grado de interacción con los ciudadanos, es decir, qué predisposición tienen a responder a sus cuestiones y fomentar el diálogo con ellos y su participación.

En el caso de Facebook, se observa que sólo el 47,62 por ciento del total de perfiles analizados tienen activada la opción de «enviar mensajes» y el 52,38 por ciento la de valorar la institución, unos porcentajes que son algo mejores entre las cuentas españolas, con un 50 por ciento y 83,33 por ciento respectivamente. Por su parte, en el caso de Twitter se observa que los perfiles de la Policía Nacional y la Guardia Civil priorizan la difusión de mensajes al diálogo con los ciudadanos. De entre todos los tuis que publican, como se apunta en el gráfico 3, sólo el uno por ciento de ellos son respuestas a usuarios. Están muy lejos en este parámetro de policías como la Bundespolizei alemana, en la que el 40 por ciento de sus mensajes son respuestas. Sí hay que destacar, en cualquier caso, que la segunda posición en este ranking está ocupada por la Policía Foral de Navarra, con un 35 por ciento.

Gráfico 3. Porcentaje de tuits que son respuestas a ciudadanos.

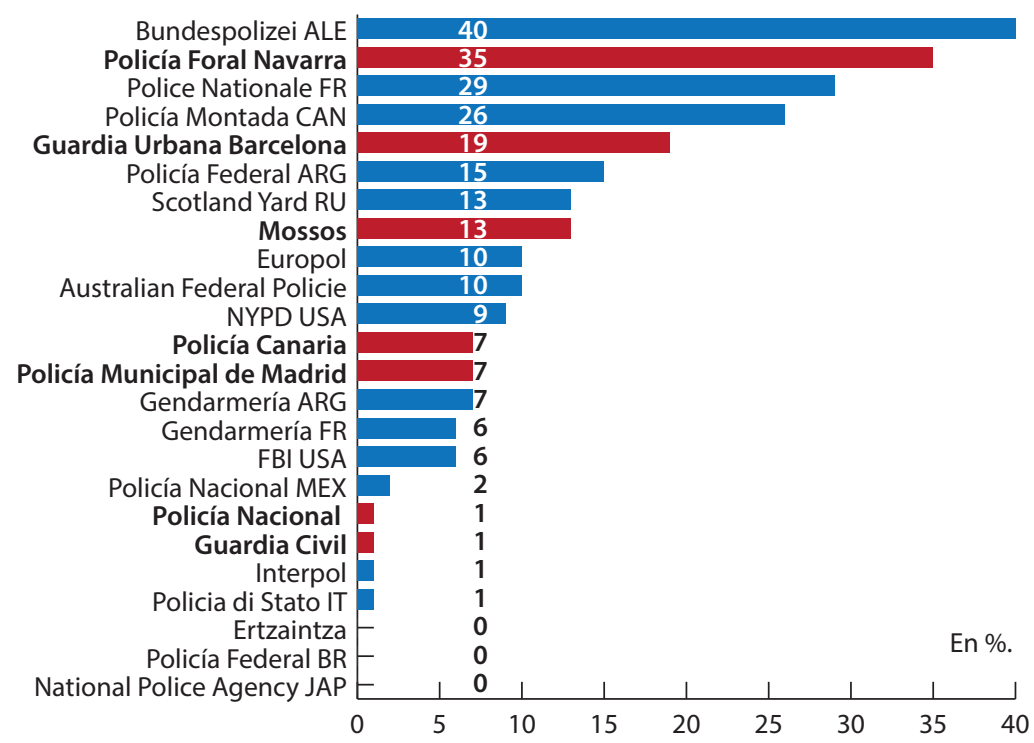

Fuente: elaboración propia a partir de la monitorización de perfiles en Twitonomy (del 1/01/2018 al 31/12/2018).

\section{Conclusiones y futuras líneas de investigación}

El presente trabajo ha tratado de realizar una primera aproximación al uso de las redes sociales por parte de los cuerpos policiales españoles en perspectiva internacional, que aún no había sido acometida con anterioridad. A la vista de los 
datos expuestos, se podría afirmar que las fuerzas y cuerpos de seguridad españoles han ido avanzando en el progresivo cumplimiento de las tres etapas descritas por Mergel (2013) y se han convertido en un caso de éxito en la aplicación de la comunicación 2.0 entre este tipo de cuerpos policiales a nivel internacional.

Han apostado decididamente, y con carácter pionero, por el uso de las redes sociales como herramienta de difusión de información, publicando más mensajes que la media de las policías internacionales analizadas. Pero no se han quedado únicamente en esta función «representativa» o de comunicación unidireccional, en la que aún permanecen muchas instituciones, sino que han ido dando pasos hacia los modelos de «compromiso» y "colaboración» con los ciudadanos. Han ido buscando así (y consiguiendo) su progresiva implicación.

El análisis cuantitativo realizado en este trabajo permite comprobar que han logrado que sus perfiles cuenten con amplias audiencias, lo que muestra un alto nivel de interés por sus publicaciones y que sus seguidores se muestren comprometidos en la difusión de las mismas y, con ello, en la amplificación de su repercusión entre la opinión pública. Se puede afirmar, por tanto, que han ganado la confianza de sus públicos, de tal forma que las redes sociales pueden seguir contribuyendo a esa buena valoración popular que muestran estos cuerpos policiales en los sondeos de opinión. En cualquier caso, y a pesar de este éxito, se ha comprobado que aún presentan cierto margen de mejora en el nivel de respuesta que dan a las peticiones de información ciudadana.

Entendemos, no obstante, que esta primera aproximación debe complementarse con futuros estudios de carácter más cualitativo que permitan comprobar cómo son los mensajes que difunden en estas redes (tanto en el fondo como en la forma) y que les han reportado el éxito descrito.

Sobre este punto, y de cara a esas futuras investigaciones, convendrá profundizar en el nuevo modelo comunicativo que han introducido en este ámbito, iniciado por la cuenta de Twitter de la Policía Nacional, que suele ser considerada como pionera en la nueva forma de comunicar de las entidades de seguridad en las redes sociales. Tal como ha narrado el que fuera primer community manager de esta cuenta, Fernández Guerra (2014), el estilo habitual en este tipo de cuentas estaba marcado por la excesiva seriedad, formalidad y burocracia. Sin embargo, la estrategia fue buscar un lenguaje más desenfadado, sencillo y emocional (e incluso atrevido y provocador en determinados momentos), tratando de llamar la atención para así conectar con todos los públicos, mediante el uso de anécdotas, chistes, gifs, emoticonos, imágenes y vídeos, memes y referencias a canciones, series y películas, etc. En definitiva, adaptándose a los códigos y el lenguaje que triunfan en las redes sociales. 
A pesar de las dudas (y críticas) iniciales que suscitó este nuevo lenguaje, la realidad es que pronto se comprobó su éxito a la hora de lograr el respaldo de los internautas, haciendo que la cuenta acumulara millones de seguidores y se convirtiera en muy poco tiempo en el cuerpo de seguridad más seguido en Twitter en todo el mundo. Esto le valió numerosos premios y reconocimientos pero, sobre todo, el que fuera tomada como referencia para otras muchas cuentas de seguridad, tanto nacionales como internacionales, que empezaron a usar también su mismo estilo de comunicación (Tosas, 2014).

Pero además de estas cuestiones formales relacionadas con el tipo de lenguaje, la novedad llegó también al ámbito de los contenidos. Como ha descrito Xesteira (2013), la cuenta de Twitter de la Policía Nacional puso de moda en otros cuerpos el difundir no sólo noticias institucionales o alertas sino también consejos de seguridad y campañas para concienciar a la población en temas como el acoso escolar, la violencia de género, el tráfico de drogas o la explotación sexual, entre otros. Asimismo, popularizó el solicitar a través de las redes la colaboración ciudadana para la resolución de delitos (como las célebres «tuit-redadas»), con un muy alto índice de participación.

La observación empírica de los perfiles en redes sociales realizada para esta investigación nos ha permitido comprobar de una forma aún muy superficial que este estilo comunicativo se ha impuesto a día de hoy en casi todas las redes sociales de organismos policiales de todo el mundo, lo que ha podido influir muy directamente en los altos números de seguidores y de difusión de sus mensajes que hemos apuntado en páginas anteriores, al tratarse de un lenguaje que conecta mucho más con los ciudadanos.

Por tanto, creemos que la academia deberá seguir profundizando en el análisis cualitativo de los mensajes que difunden a través de sus redes sociales, con el objetivo de confirmar empíricamente estos planteamientos. Además, habrá de investigar también si este modelo puede ser sostenible a medio y largo plazo en términos de éxito comunicativo, habida cuenta de que el impacto que han conseguido hasta ahora puede que vaya diluyéndose en el tiempo conforme la gente se acostumbre a ese estilo más desenfadado; sobre todo en un contexto en el que, debido a su éxito, son ya muchas las cuentas que les han imitado y, por tanto, les resulta más difícil destacar. Esto pone el foco en la necesidad de no acomodarse con el éxito conseguido y seguir contando con un enfoque estratégico, en el que se siga potenciando la creatividad pero también la investigación sobre cómo evolucionan las audiencias, qué es lo que esperan, cuáles son las nuevas tendencias narrativas (como, por ejemplo, las stories) y cómo conseguir mayor impacto para sus mensajes. A este objetivo puede contribuir también la academia, analizando ese posible cansancio del 
público hacia los contenidos y apuntando nuevos estilos, códigos y lenguajes que pueden seguir ahondando en su éxito comunicativo.

\section{Bibliografía}

AlcÁzAr Sirvent, I. (2001). Utilización de las nuevas tecnologías en la comunicación hacia la sociedad. Cuadernos de la Guardia Civil, Revista de seguridad pública, 24, 47-56. Recuperado desde: https://bit.ly/2YrkkxU

Blanco Navarro, J. M. (2017). Valoración social de la Guardia Civil. Madrid: Centro de Análisis y Prospectiva de la Guardia Civil.

Brainard, L. y EdLINS, M. (2014). Top 10 U.S. municipal police departments and their social media usage. The American Review of Public Administration, 45(6), 728-745. Doi: https://doi.org/10.1177/0275074014524478

Bullock, K. (2018). The police use of social media: transformation or normalisation? Social Policy and Society, 17(2), 245-258. Doi: https://doi.org/10.1017 \$1474746417000112

CANCElo San Martín, M. M. (2004). La comunicación en instituciones públicas: el caso de la Guardia Civil y el Cuerpo Nacional de Policía. Málaga: Ayuntamiento de Málaga.

Cancelo San Martín, M. M. (2006). La comunicación en las Fuerzas de Seguridad del Estado. Chasqui: Revista Latinoamericana de comunicación, 94, 66-71. Recuperado desde: https://bit.ly/2FxYOK

CARPentier-Laberge, C. (2015). La police et Twitter: l'utilisation des medias sociaux par les services policiers canadiens. Montreal: Université de Montréal.

Chermak, S. y Weiss, A. (2005). Maintaining legitimacy using external communication strategies: an analysis of police-media relations. Journal of Criminal Justice, 33(5), 501-512. Doi: https://doi.org/10.1016/j.jcrimjus.2005.06.001

Crump, J. (2012). What are the police doing on Twitter? Social media, the police and the public. Policy \& Internet, 2(4), 1-27. Doi: https://doi.org/10.2202/1944. 2866.1130

Cuesta, U.; Canel, M. J. y Gurrionero, M. G. (eds.) (2012). Comunicación y terrorismo. Madrid: Tecnos.

DAI, M. et al. (2017). Working with communities on social media: varieties in the use of Facebook and Twitter by local police. Online Information Review, 41(6), 782-796. Doi: https://doi.org/10.1108/OIR-01-2016-0002

Davis, E. F.; Alves, A. A. y Sklansky, D. A. (2014). Social media and police leadership. Washington D. C.: New Perspectives in Policing Bulletin.

Díez Nicolás, J. (1989). La imagen pública de la Guardia Civil. Cuadernos de la Guardia Civil, 1, 81-87. 
Díez Nicolás, J. (2012). La imagen de los servicios de inteligencia en la sociedad. Inteligencia y Seguridad, revista de análisis y prospectiva, 11, 149-160.

EdLins, M. y Brainard, L. A. (2016). Pursuing the promises of social media? Changes in adoption and usage of social media by the top 10 US police departments. Information Polity, 21(2), 171-188.

Fernández Guerra, C. (2014). @policia: las historias de un éxito. Madrid: Aguilar.

FERnÁndez VÁzQuez, J. (2014). La Guardia Civil como fuente de información. Sphera Publica, número especial del mes de junio, 64-81. Recuperado desde: https://bit.ly/2Fx3HY月

Grimmelikhuijsen, S. G. y Meijer, A. J. (2015). Does Twitter increase perceived police legitimacy? Public Administration Review, 75(4), 598-607. Doi: https://doi.org/10.1111/puar.12378

HEVERIN, T. y ZACH, L. (2011). Twitter for city police department information sharing. Proceedings of the American Society for Information Science and Techonology banner, 47(1), 1-7. Doi: https://doi.org/10.1002/meet.14504701277

Heverin, T. y ZACH, L. (2012). Law enforcement agency adoption and use of Twitter as a crisis communication tool. En HAGAR, C. (ed.). Crisis Information Management (pp. 25-42). Nueva York: Chandos Publishing. Doi: https://doi.org/10.1016/B978-1-84334-647-0.50002-3

JusTeL, M. (1982). Imagen pública de la policía. REIS, 18, 85-111. Recuperado desde: https://bit.ly/2UV0oRK

Kelly, A. y Finlayson, A. (2015). Can Facebook save neighbourhood watch? The Police Journal, 88(1), 65-77. Doi: https://doi.org/10.1177/0032258X15570557

Kingshott, B. F. (2011). Effective police management of the media. Criminal Justice Studies, 24(3), 241-253. Doi: https://doi.org/10.1080/1478601X.2011.592728

Kudla, D. y ParnabY, P. (2018). To serve and to tweet: an examination of policerelated Twitter activity in Toronto. Social Media + Society, julio-septiembre, 1-13.Doi: https://doi.org/10.1177/2056305118787520

Lee, M. y McGovern, A. (2013). Force to sell. Policing the image and manufacturing public confidence. Policing and Society, 23(2), 103-124. Doi: https://doi.org/10.1080/10439463.2011.647913

LeE, M. y McGovern, A. (2014). Policing and media: public relations, simulations and communications. Nueva York: Routledge.

Lieberman, J. D.; Koetzle, D. y Sakiyama, M. (2013). Police departments' use of Facebook: patterns and policy issues. Police Quarterly, 16(4), 438-462. Doi: https://doi.org/10.1177/1098611113495049

Lorenzo SolÁ, F. (2013). Las Relaciones Públicas en la estrategia de comunicación de la Guardia Civil de Alicante: aplicación de modelos conduc- 
tuales, tesis doctoral. Alicante: Universidad de Alicante. Recuperado desde: https://bit.ly/2CGpk8k

LuCAs-Torres, C. (2019). Policía, Guardia Civil y Ejército, las instituciones mejor valoradas. El Español, 4 de enero. Recuperado desde: https://www.elespanol.com/espana/politica/20190104/policia-guardia-civil-ejercito-instituciones-valoradas-podemos/365714320_0.htm

MawBY, R. C. (2002). Policing images: policing, communications, and legitimacy. Portland: Willan Publishing.

Mawby, R. C. (2010). Police corporate communications, crime reporting and the shaping of policing news. Policing and Society, 20(1), 124-139. Doi: https://doi.org/10.1080/10439461003611526

MeIJeR, A. J. y ToRenVLIED, R. (2016). Social media and the new organization of government communications: an empirical analysis of Twitter usage by the Dutch police. The American Review of Public Administration, 46(2), 143-161. Doi: https://doi.org/10.1177/0275074014551381

Mergel, I. (2013). A framework for interpreting social media interactions in the public sector. Government Information Quarterly, 30(4), 327-334. Doi: http:ll dx.doi.org/10.1016/j.giq.2013.05.015

Motschall, M. y CaO, L. (2002). An analysis of the public relations role of the police public information officer. Police Quarterly, 5(2), 152-180. Doi: https://doi.org/10.1177/109861102129198084

Moya Losada, E. y Blanco Navarro, J. M. (2015). Redes sociales y seguridad ciudadana: casos de éxito. En: Criado, J. I. y Rojas Martín, F. (eds.). Casos de éxito en redes sociales digitales de las administraciones públicas ( $\mathrm{pp}$. 130-154). Barcelona: Escola d'Administració Pública de Catalunya. Recuperado desde: https://bit.ly/1vcO8Hs

O'Connor, C. D. (2017). The police on Twitter: image management, community building, and implications for policing in Canada. Policing \& Society, 27(8), 899-912. Doi: https://doi.org/10.1080/10439463.2015.1120731

Olmos Hidalgo, D. (2016). Historia de las Fuerzas y Cuerpos de Seguridad del Estado en España. Valladolid: Universidad de Valladolid. Recuperado desde: hittps://bit.ly/20uAH8k

Padilla Castillo, G. (2016). Redes sociales institucionales de éxito: los casos de@policia y@guardiacivil en España. Opción, 12, 85-109. Recuperado desde: hhttps://bit.ly/2JFcMUL

Pintado Pagán, M. (2016). Las policías autonómicas en España: situación actual y perspectiva de futuro en el Estado Autonómico, tesis doctoral. Murcia: Universidad Católica San Antonio de Murcia. Recuperado desde: https://bit.ly/2HHGJ4R 
PRocter, R. (2013). Reading the riots: what were the police doing on Twitter? Policing and Society, 23(4), 413-436. Doi: https://doi.org/10.1080/10439463.2013.780223 Puig, J. J. (1984). Historia de la Guardia Civil. Barcelona: Mitre.

Raldúa Martín, E. V. (1996). Cambios en la imagen pública de la Policía (1980-1995) y situación actual. REIS, 74, 327-341. Recuperado desde: https://bit.ly/2UWMTkk

Rodrigo Alsina, M. (1991). Los medios de comunicación ante el terrorismo. Barcelona: Icaria.

RodRíGUEZ ANDRÉs, R. (2017). ¿En camino hacia el gobierno 2.0?: análisis del uso de redes sociales por los ministerios españoles para fomentar la transparencia y la participación ciudadana. Estudios sobre el Mensaje Periodístico, 23(2), 937-954. Doi: http://dx.doi.org/10.5209/ESMP.58025

Rodríguez Andrés, R. y Álvarez SABAlegui, D. (2018). Presencia y actividad de los parlamentos autonómicos en las redes sociales: pocos avances en el fomento de la participación ciudadana. El profesional de la información, 27(5), 994-1.003. Doi: https://doi.org/10.3145/epi.2018.sep.04

Ruddell, R. y Jones, N. (2013). Social media and policing: matching the message to the audience. Safer Communities, 12(2), 64-70. Doi: https://doi.org/10.1108/17578041311315030

SÁinZ, J. (2018). Policía, Ejército y Monarquía, las instituciones mejor valoradas. El Español, 3 de enero. Recuperado desde: https://www.elespanol.com/espana/politica/20180103/policia-ejercito-monarquia-instituciones-valoradas-partidos-congreso/274223162_0.htm|

SCHNEIDER, C. J. (2016). Police presentational strategies on Twitter in Canada. Policing \& Society, 26(2), 129-147. Doi: https://doi.org/10.1080/10439463.2014.922085

Schneider, C. J. y Altheide, D. L. (2016). Policing and social media. Maryland: Lexington Books.

Silvela Díaz-Criado, E. (2017). Comunicación estratégica: origen y evolución del concepto. En: VV.AA., La comunicación estratégica (pp. 13-34). Madrid: Instituto Español de Estudios Estratégicos. Recuperado desde: hittps://bit.ly/2U6sr3z Tosas, G. (2014). La cuenta de Twitter de la Policía, un caso de éxito. La Vanguardia, 20 de enero. Recuperado desde: https://www.lavanguardia.com/tecnologia redes-sociales/twitter/20140120/54398228799/twitter-policia-caso-exito.htm

Trottier, D. (2012). Policing social media. Canadian Review of Sociology, 49(4), 411-425. Doi: https://doi.org/10.1111/j.1755-618X.2012.01302.x

Turrado Vidal, M. (2000). Historia del Cuerpo Nacional de Policía. Cuadernos de la Guardia Civil, 22, 183-204. Recuperado desde: https://bit.ly/20q57se

Twittter (2019). Ranking de twitteros más seguidos en España (51 al 100). TwitterEspaña.com, 23 de marzo. Recuperado desde: http://twitter-espana.com/ranking/top-100, 
Van de Velde, B.; Meijer, A. y Homburg, V. (2015). Police message difussion on Twitter: analysing the reach of social media communications. Behaviour \& Information Technology, 34(1), 4-16. Doi: https://doi.org/10.1080/0144929X.2014.942754 Wyво, J. L. et al. (2014). L'impact des réseaux sociaux dans la gestión des crises et le cybercrime. Sécurité et stratégie, 18(3), 37-43. Doi: https://doi.org/10.3917/sestr.018.0037 Xesteira, M. (2013). Las 35 claves del éxito de la cuenta de Twitter de la Policía Nacional. MiltonFactory, 18 de junio. Recuperado desde: https:/les.slideshare.net MiltonFactory/las-35-claves-del-xito-de-la-cuenta-de-twitter-de-la-polica-nacional

Para citar este artículo: Rodríguez-Andrés, R. y López-García, J. M. (2019).

Aproximación al uso de las redes sociales por las fuerzas y cuerpos de seguridad en España en perspectiva internacional. index.comunicación, 9(1), 127-148. 